\title{
Long-Term, All-Day Exposure to Circadian-Effective Light Improves Sleep, Mood, and Behavior in Persons with Dementia
}

\author{
Mariana G. Figueiro ${ }^{\mathrm{a}, *}$, Levent Sahin ${ }^{\mathrm{a}}$, Michael Kalsher ${ }^{\mathrm{b}}$, Barbara Plitnick ${ }^{\mathrm{a}}$ and Mark S. Rea ${ }^{\mathrm{a}}$ \\ ${ }^{a}$ Lighting Research Center, Rensselaer Polytechnic Institute, Troy, NY, USA \\ ${ }^{\mathrm{b}}$ Department of Cognitive Science, School of Humanities, Arts and Social Sciences, Rensselaer Polytechnic \\ Institute, Troy, NY, USA
}

\begin{abstract}
.
Background: Persons with Alzheimer's disease and related dementias (ADRD) frequently experience sleep-wake (circadian) cycle disturbances that lead them to remain awake at night, causing stress and fatigue for families and caregivers. Light therapy shows promise as a nonpharmacological treatment for regulating sleep in this population.

Objective: We investigated the long-term impact of a circadian-effective lighting intervention on sleep, mood, and behavior problems in persons with ADRD.

Methods: This 25-week clinical trial administered an all-day lighting intervention to 47 patients with ADRD in 9 senior-care facilities, employing wrist-worn actigraphy measures and standardized measures of sleep quality, mood, and behavior.

Results: The intervention significantly improved Pittsburgh Sleep Quality Index scores, from an estimated mean \pm SEM of $11.89 \pm 0.53$ at baseline to $5.36 \pm 0.63$ at the end of the intervention. Additional improvements were noted for sleep efficiency data from actigraph measurements. The intervention significantly reduced Cornell Scale for Depression in Dementia scores (mean \pm SEM of $11.36 \pm 0.74$ at baseline and $4.18 \pm 0.88$ at the end of the intervention) and Cohen-Mansfield Agitation Inventory scores (mean \pm SEM of $47.10 \pm 1.98$ at baseline and $35.33 \pm 2.23$ at the end of the intervention).

Conclusion: A regular circadian-effective daytime lighting intervention can improve sleep at night and reduce depression and agitation in patients with dementia living in controlled environments. More importantly, the positive effects of the tailored lighting intervention on these outcomes appear to be cumulative over time.
\end{abstract}

Keywords: Alzheimer's disease and related dementias, circadian system, lighting intervention, mood, rest-activity, sleep

\footnotetext{
${ }^{*}$ Correspondence to: Mariana G. Figueiro, PhD, Lighting Research Center, Rensselaer Polytechnic Institute, 21 Union Street, Troy, NY 12280, USA. Tel.: +1 518276 7142; E-mail: figuem@rpi.edu.
}

\section{INTRODUCTION}

Of the estimated 5.8 million people in the United States living with Alzheimer's disease [1] and related dementias (ADRD), at least one-third experience difficulty sleeping $[2,3]$ and approximately two-thirds of their estimated 18.5 million unpaid caregivers 
report sleep disturbances themselves $[1,4,5]$. The precipitating factor for institutionalization of those with ADRD is often a disturbed sleep-wake (circadian) cycle that leads them to remain awake at night, causing stress and fatigue for their families and caregivers. This behavior continues in nursing home environments, where residents with daytime agitation behavior also tend to sleep poorly at night and nap during the day [6].

Compared to healthy older adults, persons with ADRD demonstrate lower sleep efficiency and morefrequent nighttime arousals, with the severity of their sleep disturbance paralleling the level of the disease $[7,8]$. Several mechanisms have been postulated for the prevalence of sleep disorders in ADRD, the foremost being a reduction in neuronal receptiveness to environmental cues of light and dark due to the degeneration of the optic nerve and retinal ganglion cells $[9,10]$, the loss of functionality of the "biological clock" located in the suprachiasmatic nucleus [11, 12], and the exacerbation of the preceding two factors by inadequate exposure to bright light compared to healthy elderly controls [13, 14]. Moreover, changes to the aging eye, such as reduced pupil size (pupillary miosis) and lens thickening, result in a reduction of retinal illumination by as much as two-thirds in older persons compared to young adults [15]. These changes also progressively reduce the transmission of short-wavelength light to the retina, resulting in a concurrent reduction of circadian-effective light on the retina, and thus, a greater risk of circadian disruption in aging adults [16]. Physiological studies have demonstrated fragmented circadian rhythms, circadian phase delays, and diminished regularity of circadian body temperature and hormonal cycles in persons with ADRD [17-19].

Although widely used, little is known about the safety and effectiveness of medications for treating chronic sleep disturbances in persons with ADRD. Studies have shown that prescription drugs do not improve overall ratings of sleep quality in older adults, including those with ADRD $[20,21]$. The problems associated with sleep-inducing medications for older people who are cognitively impaired include increased risk for falls, fractures, and overdose; increased confusion; and an overall decline in the ability to care for oneself [22-24]. Furthermore, there are ongoing financial and practical burdens associated with obtaining prescriptions, purchasing sleep medications, and arranging the supervision of their administration [1], as well as the possibility of unintended negative side effects resulting from the combination of sleep medications with other prescriptions [25]. The treatment benefits of using sleep medications in older individuals with ADRD may not outweigh the potential risks [21].

Light therapy has shown great promise as a nonpharmacological treatment to help regulate sleep in ADRD patients. Studies have demonstrated that daytime light exposure can consolidate and increase nighttime sleep efficiency while increasing daytime wakefulness and reducing evening agitation [26, 27]. More importantly, daytime light exposure has been shown to improve memory and cognition in ADRD patients [28]. Longer and better nighttime sleep should diminish disruptive behaviors in people with ADRD during the day and, by extension, have a positive impact on caregivers, both in institutions and at home.

The circadian clock, and thus the timing of sleep, responds best to short-wavelength light; nocturnal melatonin suppression (a well-established marker of the circadian system) has a peak spectral sensitivity near $460 \mathrm{~nm}[29,30]$. Light sources typically used in elder care facilities, however, do not necessarily provide enough short-wavelength stimulation to the circadian system. In a recent 4-week field study conducted in eight long-term care facilities, we demonstrated that a daytime lighting intervention designed to maximally affect the circadian system improved patients' sleep quality and reduced depression and agitation scores relative to a control lighting condition delivering low levels of circadian-effective light [31]. The successful lighting intervention was not provided by conventional ceiling mounted luminaires, but rather by tailored lighting intervention (TLI) devices that matched the behavior patterns of the patients and thereby provided circadian-effective light that actually reached their eyes. It remained unknown, however, whether an intervention of longer duration would accrue greater benefit or result in an adaptation to the stimulus.

To date, only one study has investigated the long-term (maximum $=3.5$ years, mean $=15$ months) impact of a lighting intervention delivering very high levels of white light in common spaces of elder care facilities, where patients spent most of their daytime hours [28]. That study demonstrated that high levels (1000lx) of "white" light improved sleep and cognition in persons with ADRD. The disadvantage of the lighting intervention used in that study, however, was that the light levels were 10 times higher than those normally found in such facilities, which would increase energy use and could cause visual 
discomfort. Tailoring the light actually reaching the eyes to the spectral and absolute sensitivities of the circadian system, on the other hand, would permit maximum effectiveness while minimizing energy use and the risk of glare.

The primary aim of present study was to extend our earlier findings $[26,31,32]$ by determining whether a long-term, 24-week TLI delivering a high level of circadian stimulation during the day would improve subjective and objective measures of nighttime sleep in persons with ADRD living in controlled environments. The secondary aim was to determine whether the TLI would improve caregiver-assessed participant scores in measures of depression, agitation, and quality of life in the same population. We hypothesized that, compared to baseline lighting conditions, a longer term exposure than the duration used in our previously investigated TLI would result in greater, longer-lasting improvements to sleep, mood, and behavior.

\section{METHODS}

\section{Participant selection}

Participants were recruited from three assistedliving facilities (each with a dedicated memory-care units) and six long-term care facilities (three with dedicated memory-care units) in the New York Capital District, South Bend, IN, and Syracuse, NY. All participants had private bedrooms but spent the majority of the daytime hours in common areas supervised by facility caregivers. Rolling recruitment for the study began in August 2014 and continued through February 2019. Potential study participants $(\mathrm{N}=80)$ identified by the facility nurse or physician as having sleep problems were screened by research staff and informed consent was obtained from the responsible family members. Fifty-one participants who satisfied the study's inclusion and exclusion criteria were enrolled, and 47 participants (mean $[\mathrm{SD}]=85.3$ years $[7.1]$; $57.4 \%$ were female) underwent the study's outcome measurements at baseline and at least one data collection week during the lighting intervention (Table 1).

The study was conducted in accordance with the Declaration of Helsinki [33] and was approved by the Institutional Review Board at Rensselaer Polytechnic Institute. There was minimal risk of harm to the participants, as no known safety risks are associated with the devices used in the study and all comply
Table 1

Characteristics of participants

\begin{tabular}{lc}
\hline Characteristic & Total \\
\hline Participants, $N(\%)$ & $47(100)$ \\
Residing in assisted living facility, No. $(\%)$ & $17(36.2)$ \\
Residing in long-term care facility, No. (\%) & $30(63.8)$ \\
Female, $n(\%)$ & $27(57.4)$ \\
Mean (SD) age & $88.8(6.2)$ \\
Mean (SD) MMSE score & $15.2(3.4)$ \\
Mean (SD) BIMS score & $4.0(1.7)$ \\
Male, $n$ (\%) & $20(42.6)$ \\
Mean (SD) age & $80.7(5.4)$ \\
Mean (SD) MMSE score & $13.6(2.9)$ \\
Mean (SD) BIMS score & $5.6(2.9)$ \\
\hline
\end{tabular}

MMSE, Mini-Mental State Examination; BIMS, Brief Interview for Mental Status.

with federal regulations regarding electromagnetic and radio interference.

Inclusion criteria required a diagnosis of dementia according to Diagnostic and Statistical Manual of Mental Disorders, Fourth Edition [34]; a MiniMental State Examination (MMSE) [35] score between 4 and 24 points (indicating severe $[<12$ points] to mild [ $<24$ points] dementia) or a Brief Interview for Mental Status (BIMS) [36] score between 3 and 12 points (indicating severe $[<7$ points] to moderate [8-12 points] cognitive impairment), depending on the particular instrument employed in the facility's evaluation procedures; and a score $>5$ points (indicating sleep disturbance) on the Pittsburgh Sleep Quality Index (PSQI) questionnaire [37]. While not identical, the MMSE and BIMS measures are highly correlated [38].

Participants were excluded from the study if they had major organ failure, a major illness, a history of head injury, uncontrolled generalized disorders (e.g., diabetes), obstructing cataracts, macular degeneration, blindness, or used psychotropic medicine. Those with severe sleep apnea or restless legs syndrome were also excluded. Sleep apnea was screened using the Sleep Apnea scale of the Sleep Disorders Questionnaire [39], with a cutoff score of 29 points for men (sensitivity $75 \%$, specificity $65 \%$ ) and 26 points for women (sensitivity $80 \%$, specificity $67 \%$ ). Restless legs syndrome was screened using the International Restless Legs Syndrome Study Group rating scale [40], with a cutoff score $\geq 11$ points (indicating at least moderate symptoms) for all participants. Co-morbidities of participants included hypertension, depression, anxiety, hypothyroidism, atrial fibrillation, gastroesophageal reflux disease, and hyperlipidemia. No exclusions were made based on medication use except the use of psychotropic 
(sleep aid) medicine. Participants using antidepressant medications were included but physicians were asked not to change participants' dosages during the study period; no changes were observed nor reported.

\section{Tailored lighting intervention (TLI)}

Participants were exposed to a single daytime $(\approx 06: 00-08: 00$ to $18: 00)$ TLI that provided high levels of circadian-effective light. The light delivery method (i.e., custom-built floor lamps, light boxes, and light tables) varied depending on where individual participants spent most of their day (Supplementary Table 1). Briefly, the TLI was designed to deliver a targeted level (0.4) of circadian stimulus (CS) [41-43]. Circadian light $\left(\mathrm{CL}_{\mathrm{A}}\right)$ is irradiance weighted by the spectral sensitivity of the retinal phototransduction mechanisms stimulating the response of the biological clock, based on nocturnal melatonin suppression. $\mathrm{CS}$ is a transformation of $\mathrm{CL}_{\mathrm{A}}$ into a relative scale from approximately $0.1(\approx 10 \%)$, the threshold for circadian system activation, to approximately $0.7(\approx 70 \%)$, response saturation, and is equivalent to nocturnal melatonin suppression in percent after a 1-hour exposure to light.

On the day of the lighting installation, light levels were measured at participants' eyes via spectroradiometer (model BTS256-E, Gigahertz-Optik, Amesbury, MA). A custom software application was developed to allow for real-time photopic illuminance and CS measurements. Window shades were closed during measurement to isolate the contribution of circadian-effective light by the TLI interventions. If the window shades could not be closed, the space's luminaires were not energized and a measurement was taken to account for any additional light from sources other than the intervention or control light.

Timers controlled all lights for the TLI, activating the lights according to individual participants' habitual wake times (generally $\approx 06: 00-08: 00$ ). The lights were placed in participants' bedrooms or in common areas (e.g., dining room) for participants who spent most of the day outside their bedrooms. The existing facility lighting, delivering a $\mathrm{CS}<0.1$ at eye level, was used in all spaces after 18:00.

\section{Field monitoring procedures and analyses}

\section{Circadian stimulus (CS)}

During the data collection weeks, each participant wore a Daysimeter [44] device as a pendant (i.e., at chest height) during waking hours and placed the device next to their bed during sleep. The Daysimeter is a small device that continuously records light exposures (using a red-green-blue [RGB] solid-state photosensor package) and activity levels. Caregivers were instructed to ensure that participants did not cover the device with blankets or garments. Upon downloading, the RGB values were converted into values of CS [41-43] and into values photopic illuminance, the common measure of light in architectural spaces [45]. In order to minimize the impact of skewed data (e.g., a brief increase in $\mathrm{CL}_{\mathrm{A}}$ due to sunlight hitting the Daysimeter's sensor), geometric mean values for $\mathrm{CL}_{\mathrm{A}}$ were calculated for transformation into the CS values used in the analysis.

\section{Questionnaires}

Four questionnaires were completed by nighttime caregivers to assess participants' subjective sleep quality, depression, agitation, and quality of life. The primary outcome measure was the PSQI because it addressed sleep quality [37]. The PSQI is a tool for measuring sleep quality in clinical populations. It is composed of 19 items that generate seven component scores (subjective sleep quality, sleep latency, sleep duration, habitual sleep efficiency, sleep disturbances, use of sleep medication, and daytime dysfunction). The sum of the seven component scores yields one global score. Global scores $>5$ points indicate sleep disturbances.

Secondary outcome measures included the Cornell Scale for Depression in Dementia (CSDD) [46], the Cohen-Mansfield Agitation Inventory (CMAI) [47], and the Minimum Data Set Activities of Daily Living Scale (MDS-ADL) [48].

The CSDD [46] is a 19-item tool that evaluates the presence and extent of mood-related signs, behavioral disturbances, physical signs, cyclic functions, and ideational disturbances. The items are measured on a $0-3$ point scale, with total scores $>12$ indicating probable major depression.

The CMAI [47] assesses the frequency of manifestations of agitation behavior observed in participants by caregivers. The CMAI consists of 29 agitation behaviors, each rated on a 7-point scale of frequency. Higher CMAI scores indicate greater frequency of agitation, with scores $>45$ indicating clinically significant agitation.

The MDS-ADL [48] measures activities related to personal care and includes bathing, dressing, getting in or out of bed or a chair, using the toilet, and eating. Scores range from zero (total independence or no or little help with an activity) to four (total dependence, 
full staff participation in activity during the entire week). A higher total score is associated with greater dependence in the performance of personal care.

\section{Actigraphy}

Participants wore an actigraph (Actiwatch 2, Philips Respironics, Murraysville, PA) on their nondominant wrist that recorded rest-activity rhythms for the calculation of interdaily stability (IS) and intradaily variability (IV) [49] as primary outcomes. IS is a ratio that quantifies the extent to which all recorded 24-hour activity profiles resemble each other, which represents the day-by-day regularity of the sleep-wake pattern. Higher IS ratios indicate better interdaily stability. IV is a ratio that quantifies the fragmentation of the rhythm, or the frequency and extent of transitions between periods of rest and activity. Lower IV ratios indicate better intradaily variability. The actigraphy data were also used to obtain estimates of sleep parameters, including actual sleep time (the amount of time between sleep start and sleep end that is scored as sleep according the actigraph software), actual sleep time percentage, actual wake time (the amount of time between sleep start and sleep end that is scored as wake according the actigraph software), actual wake time percentage, sleep efficiency (percentage of actual sleep between sleep onset and final waking), sleep onset latency (the amount of time between lights out and sleep onset), and daytime naps. Actigraphy data were analyzed using Philips Respironics Actiware software (version 6.0.9).

\section{Study protocol}

This within-subjects field study was conducted over a 6-month period (Fig. 1). The TLI commenced upon completion of a 1-week baseline assessment, and data were collected during weeks 3,9,17, and 25 of the study (see Fig. 1). The facility's night-shift caregiver who was most familiar with a particu- lar participant's sleep and behavior completed the questionnaires, performing all assessments. Participants wore the Daysimeter and actigraph during the data collection weeks. Research staff returned to the facility at the end of the baseline week for installation of the TLI, and again returned at the beginning of weeks $3,9,17$, and 25 to request that participants wear the Daysimeter and actigraph for the ensuing week. At the end of each respective study week, the questionnaires were administered and the devices were collected from the participants by research staff.

\section{Statistical analysis}

Participants were included in the analyses only if they had usable data for the baseline measurement and at least one data collection week during the lighting intervention. Two participants were excluded from the actigraphy analysis because they did not wear the actigraph during one or more data collection weeks. Fifteen participants did not complete all 25 weeks of the study (Supplementary Table 2).

Linear mixed-effects models (LMEMs) were constructed for data analysis using SPSS, version 24.0, for Windows (SPSS Inc., Chicago, IL). The criterion for statistical significance in all analyses was $p<0.05$. While the central focus of the study's primary and secondary aims was the efficacy of the TLI, we also assessed the statistical effects of participants' sex and level of dementia (cognitive state). For the cognitive state variable, participants were placed into one of two categories (i.e., mild-moderate dementia or severe dementia) based on their BIMS or MMSE scores, when available (see Participant Selection). (The mild and moderate categories were merged due to the small number of participants in the moderate range $[n=3])$. No BIMS or MMSE scores were available for nine participants. However, as these individuals resided in an assisted living facility where the admission criteria were limited to residents with mild-moderate dementia, explicitly excluding those

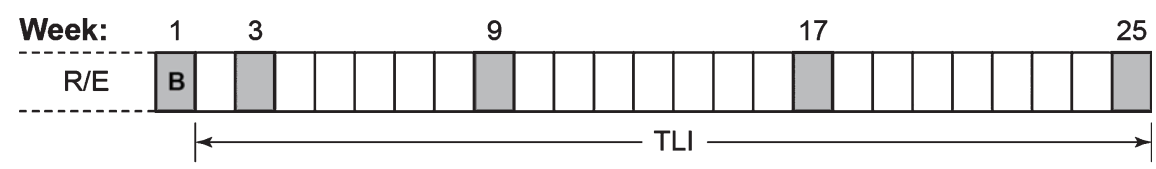

\section{Legend: R/E Recruitment/enrollment B Baseline measurement}

Data collection week (questionnaires [last day], actigraphy, Daysimeter)

Fig. 1. The study protocol. The 25 -week protocol was composed of a 1 -week baseline measurement period (week 1) and four additional data collection weeks (weeks 3, 9, 17, and 25) during the course of the tailored lighting intervention (TLI). 
determined to have severe dementia, the nine participants without BIMS or MMSE assessments were coded as mild-moderate for purposes of the data analysis.

Although initially considered for inclusion in the analysis, participant age was ultimately excluded as a variable because Pearson's product-moment correlations between age and the outcomes revealed nonsignificant results $(p>0.05)$ for all but one of the measures (CSDD at week 3 only).

Data collection week (i.e., at baseline and during the lighting intervention), sex, and cognitive state were entered into the model as fixed effects and participant was entered as a random effect. Significant effects due to main factors (i.e., data collection week, sex, and cognitive state) and/or their interactions were followed up with multiple comparisons using twosided $t$-tests with Sidak correction for Type I errors. Cohen's $d$ is reported as a measure of effect size for significant differences. Estimated mean and standard error of the mean (SEM) values are reported.

\section{RESULTS}

\section{Circadian stimulus (CS)}

In order to verify the efficacy of the TLI, unpaired $t$-tests were performed comparing measurements of CS at baseline and during weeks 3,9,17, and 25. The mean CS participants received during the intervention was significantly greater compared to baseline. CS values did not reach the target CS of 0.4 , most likely because the Daysimeter was being covered by sweater and blankets, as was observed by the research team during site visits. Site measurements using a sprectroradiometer confirmed that the TLI was delivering the target $\mathrm{CS}$ values.

\section{Primary aim}

\section{Subjective assessment of sleep quality (PSQI)}

Consistent with the study's primary aim, the LMEM for PSQI revealed a significant effect of data collection week (Table 2A). Mean PSQI scores were significantly lower at weeks $3,9,17$, and 25 compared to baseline (Fig. 2A, Supplementary Table 3). The scores were also significantly lower at weeks 9, 17, and 25 compared to week 3 . At the end of week 25, the estimated mean PSQI score was 5.36, which is virtually at the threshold score $(>5)$ indicating sleep disturbances. There was also a significant main effect of sex (see Table 2A), with males exhibiting greater sleep disturbances than females. The mean PSQI score for males (mean $\pm \mathrm{SEM}=9.09 \pm 0.62$ ) was significantly higher than that for females (mean \pm SEM $=6.93 \pm 0.53$ ).

\section{Objective assessment of sleep quality (actigraphy)}

Interdaily stability (IS) and intradaily variability $(I V)$. No significant effects were associated with IS, but the LMEM for IV revealed a significant main effect of cognitive state (see Table 2A). On average, participants with severe dementia showed significantly lower IV values (mean $\pm \mathrm{SEM}=0.95 \pm 0.07$ ) than those with mild-moderate dementia (mean \pm $\mathrm{SEM}=1.13 \pm 0.05$ ).

Actual Sleep Time. The LMEM for actual sleep time revealed significant main effects of sex and cognitive state (see Table 2A). The mean value was significantly greater for females (mean $\pm \mathrm{SEM}=516.95 \pm$ $20.55 \mathrm{~min}$ ) than for males (mean $\pm \mathrm{SEM}=448.91$ $\pm 23.43 \mathrm{~min}$ ). In addition, actual sleep time was also greater in those with mild-moderate dementia (mean $\pm \mathrm{SEM}=531.16 \pm 17.23 \mathrm{~min})$ than in those with severe dementia (mean $\pm \mathrm{SEM}=434.70 \pm 25.97$ $\min )$.

Actual sleep time percentage. The LMEM for actual sleep time percentage revealed a significant main effect of sex (see Table 2A). On average, actual sleep time percentage for females (mean \pm SEM $=93.14$ \pm 0.98 ) was significantly greater than that for males (mean \pm SEM $=89.91 \pm 1.12$ ).

Actual wake time. No significant effects were associated with this outcome measure, nor did actual wake time differ significantly across the data collection weeks.

Actual wake time percentage. The LMEM for actual wake time percentage revealed a significant main effect of sex (see Table 2B). On average, actual wake time percentage for males (mean $\pm \mathrm{SEM}=10.23 \pm$ 1.20) was significantly greater than that for females (mean $\pm \mathrm{SEM}=6.71 \pm 1.05$ ).

Sleep efficiency. The LMEM for sleep efficiency revealed a significant main effect of data collection week (see Table 2B). Sleep efficiency was significantly greater at weeks 3,17 , and 25 compared to baseline; the differences between baseline and 
Table 2A

LMEM results for the study's primary aim, by outcome measure

\begin{tabular}{|c|c|c|c|c|c|c|}
\hline Outcome & $\begin{array}{l}\text { Estimated Mean } \pm \text { SEM, } \\
\text { by week }\end{array}$ & Main effect/interaction & df & Error & $F$ & $p$ \\
\hline \multicolumn{7}{|c|}{ Subjective outcome (questionnaire) } \\
\hline \multirow[t]{7}{*}{ Sleep quality (PSQI) } & Baseline $=11.89 \pm 0.53$ & Data collection week & 4 & 98.34 & 24.39 & $<0.001$ \\
\hline & Week $3=8.74 \pm 0.53$ & Sex & 1 & 43.18 & 6.90 & 0.01 \\
\hline & Week $9=7.23 \pm 0.55$ & Cognitive state & 1 & 43.18 & 2.78 & 0.10 \\
\hline & Week $17=6.82 \pm 0.60$ & Data collection week $\times$ sex & 4 & 98.34 & 0.79 & 0.54 \\
\hline & Week $25=5.36 \pm 0.63$ & Data collection week $\times$ cognitive state & 4 & 98.34 & 1.76 & 0.14 \\
\hline & & Sex $\times$ cognitive state & 1 & 43.18 & 0.00 & 0.99 \\
\hline & & Data collection week $\times$ sex $\times$ cognitive state & 4 & 98.34 & 0.49 & 0.74 \\
\hline \multicolumn{7}{|c|}{ Objective outcomes (actigraphy) } \\
\hline \multirow[t]{7}{*}{ IS } & Baseline $=0.36 \pm 0.03$ & Data collection week & 4 & 96.90 & 1.30 & 0.28 \\
\hline & Week $3=0.35 \pm 0.03$ & Sex & 1 & 40.73 & 1.11 & 0.30 \\
\hline & Week $9=0.41 \pm 0.03$ & Cognitive state & 1 & 40.73 & 0.04 & 0.84 \\
\hline & Week $17=0.35 \pm 0.03$ & Data collection week $\times$ sex & 4 & 96.90 & 1.29 & 0.28 \\
\hline & Week $25=0.40 \pm 0.03$ & Data collection week $\times$ cognitive state & 4 & 96.90 & 0.31 & 0.871 \\
\hline & & Sex $\times$ cognitive state & 1 & 40.73 & 0.38 & 0.54 \\
\hline & & Data collection week $\times$ sex $\times$ cognitive state & 4 & 96.90 & 0.74 & 0.57 \\
\hline \multirow[t]{7}{*}{ IV } & Baseline $=1.05 \pm 0.06$ & Data collection week & 4 & 89.67 & 0.23 & 0.92 \\
\hline & Week $3=1.05 \pm 0.06$ & Sex & 1 & 39.62 & 0.06 & 0.81 \\
\hline & Week $9=1.03 \pm 0.06$ & Cognitive state & 1 & 39.62 & 4.40 & 0.04 \\
\hline & Week $17=1.07 \pm 0.06$ & Data collection week $\times$ sex & 4 & 89.67 & 0.92 & 0.46 \\
\hline & Week $25=1.01 \pm 0.06$ & Data collection week $\times$ cognitive state & 4 & 89.67 & 1.98 & 0.11 \\
\hline & & Sex $\times$ cognitive state & 1 & 39.62 & 0.22 & 0.64 \\
\hline & & Data collection week $\times$ sex $\times$ cognitive state & 4 & 89.67 & 0.17 & 0.95 \\
\hline \multirow[t]{7}{*}{ Actual sleep time } & Baseline $=473.69 \pm 18.28$ & Data collection week & 4 & 119.92 & 0.34 & 0.86 \\
\hline & Week $3=478.10 \pm 18.60$ & Sex & 1 & 42.34 & 4.77 & 0.04 \\
\hline & Week $9=481.30 \pm 18.83$ & Cognitive state & 1 & 42.34 & 9.57 & 0.003 \\
\hline & Week $17=491.00 \pm 20.30$ & Data collection week $\times$ sex & 4 & 119.92 & 0.61 & 0.66 \\
\hline & Week $25=490.56 \pm 20.31$ & Data collection week $\times$ cognitive state & 4 & 119.92 & 0.99 & 0.42 \\
\hline & & Sex $\times$ cognitive state & 1 & 42.34 & 0.35 & 0.56 \\
\hline & & Data collection week $\times$ sex $\times$ cognitive state & 4 & 119.92 & 0.89 & 0.48 \\
\hline \multirow[t]{7}{*}{ Actual sleep time percentage } & Baseline $=90.73 \pm 0.94$ & Data collection week & 4 & 113.30 & 0.69 & 0.60 \\
\hline & Week $3=91.67 \pm 0.97$ & Sex & 1 & 40.29 & 4.73 & 0.04 \\
\hline & Week $9=91.05 \pm 0.98$ & Cognitive state & 1 & 40.29 & 0.11 & 0.74 \\
\hline & Week $17=92.33 \pm 1.08$ & Data collection week $\times$ sex & 4 & 113.30 & 0.79 & 0.53 \\
\hline & Week $25=91.84 \pm 1.07$ & Data collection week $\times$ cognitive state & 4 & 113.30 & 0.85 & 0.50 \\
\hline & & Sex $\times$ cognitive state & 1 & 40.29 & 0.65 & 0.43 \\
\hline & & Data collection week $\times$ sex $\times$ cognitive state & 4 & 113.30 & 0.96 & 0.43 \\
\hline
\end{tabular}

PSQI, Pittsburgh Sleep Quality Index; IS, interdaily stability; IV, intradaily variability. Statistically significant $(p<0.05)$ results are shown in bold.

week 9 were close to significance, but not statistically significant after Sidak correction (Fig. 2B; see Supplementary Table 3).

Sleep onset latency. The LMEM for sleep onset latency revealed significant main effects of data collection week and cognitive state (see Table 2B). Post hoc comparisons after Sidak correction revealed that sleep onset latency was statistically significantly lower at week 9 compared to week 3 and week 25 (Fig. 2C; see Supplementary Table 3). Sleep onset latency was also significantly greater in participants with severe dementia than in those with mild-moderate dementia.
Daytime naps. No significant effects were associated with this outcome measure, nor did daytime naps differ significantly across the data collection weeks.

\section{Secondary aim}

Subjective assessment of depression (CSDD)

The LMEM for CSDD revealed a significant main effect of data collection week (Table 3). Mean CSDD scores were significantly lower at weeks $3,9,17$, and 25 compared to baseline (Fig. 3A, Supplementary Table 4). Mean CSDD scores were also significantly lower at week 25 compared to week 3. Lower CSDD scores indicate less depression. 



Fig. 2. Results for the study's primary aim, showing the statistically significant effect of data collection week (A-C). Compared to baseline and the first data collection week of the lighting intervention (week 3), participants' mean PSQI scores decreased (i.e., improved) significantly as the lighting intervention progressed, falling to close to the threshold for the absence of sleep disturbances (scores $<5$, dashed line) by week 25 (A). Compared to baseline, participants' mean sleep efficiency values increased significantly (i.e., improved) at data collection weeks 3 , 17, and 25 (B). Sleep onset latency decreased (i.e., improved) between data collection weeks 3 and 9 but increased between weeks 9 and 25 (C). The values represent estimated means and the error bars indicate SEM. PSQI, Pittsburgh Sleep Quality Index; ${ }^{*} p<0.05 ; * * * p<0.001$.

Table 2B

LMEM results for the study's primary aim (continued), by outcome measure

\begin{tabular}{|c|c|c|c|c|c|c|}
\hline Outcome & $\begin{array}{l}\text { Estimated Mean } \pm \text { SEM, } \\
\text { by week }\end{array}$ & Main effect/interaction & $\mathrm{df}$ & Error & $F$ & $p$ \\
\hline \multirow[t]{7}{*}{ Actual wake time } & Baseline $=45.57 \pm 5.10$ & Data collection week & 4 & 114.37 & 0.33 & 0.86 \\
\hline & Week $3=43.67 \pm 5.23$ & Sex & 1 & 38.57 & 2.37 & 0.13 \\
\hline & Week $9=47.32 \pm 5.32$ & Cognitive state & 1 & 38.57 & 1.60 & 0.21 \\
\hline & Week $17=42.04 \pm 5.89$ & Data collection week $\times$ sex & 4 & 114.37 & 0.88 & 0.48 \\
\hline & Week $25=41.19 \pm 5.88$ & Data collection week $\times$ cognitive state & 4 & 114.37 & 1.95 & 0.11 \\
\hline & & Sex $\times$ cognitive state & 1 & 38.57 & 0.66 & 0.42 \\
\hline & & Data collection week $\times \operatorname{sex} \times$ cognitive state & 4 & 114.37 & 0.59 & 0.67 \\
\hline \multirow[t]{7}{*}{ Actual wake time percentage } & Baseline $=8.92 \pm 0.99$ & Data collection week & 4 & 112.56 & 0.41 & 0.80 \\
\hline & Week $3=8.49 \pm 1.01$ & Sex & 1 & 40.35 & 4.85 & 0.03 \\
\hline & Week $9=8.97 \pm 1.02$ & Cognitive state & 1 & 40.35 & 0.27 & 0.61 \\
\hline & Week $17=7.81 \pm 1.12$ & Data collection week $\times$ sex & 4 & 112.56 & 0.76 & 0.55 \\
\hline & Week $25=8.16 \pm 1.11$ & Data collection week $\times$ cognitive state & 4 & 112.56 & 1.35 & 0.26 \\
\hline & & Sex $\times$ cognitive state & 1 & 40.35 & 0.60 & 0.44 \\
\hline & & Data collection week $\times \operatorname{sex} \times$ cognitive state & 4 & 112.56 & 1.40 & 0.24 \\
\hline \multirow[t]{7}{*}{ Sleep efficiency } & Baseline $=84.53 \pm 1.19$ & Data collection week & 4 & 99.56 & 7.92 & $<0.001$ \\
\hline & Week $3=88.04 \pm 1.22$ & Sex & 1 & 40.27 & 3.59 & 0.07 \\
\hline & Week $9=87.56 \pm 1.23$ & Cognitive state & 1 & 40.27 & 0.74 & 0.40 \\
\hline & Week $17=90.22 \pm 1.33$ & Data collection week $\times$ sex & 4 & 99.56 & 0.95 & 0.44 \\
\hline & Week $25=89.74 \pm 1.32$ & Data collection week $\times$ cognitive state & 4 & 99.56 & 1.18 & 0.32 \\
\hline & & Sex $\times$ cognitive state & 1 & 40.27 & 2.07 & 0.16 \\
\hline & & Data collection week $\times \operatorname{sex} \times$ cognitive state & 4 & 99.56 & 1.62 & 0.18 \\
\hline \multirow[t]{7}{*}{ Sleep onset latency } & Baseline $=14.08 \pm 2.84$ & Data collection week & 4 & 99.99 & 4.10 & 0.004 \\
\hline & Week $3=15.30 \pm 2.90$ & Sex & 1 & 39.30 & 2.34 & 0.13 \\
\hline & Week $9=5.91 \pm 2.96$ & Cognitive state & 1 & 39.30 & 4.14 & 0.049 \\
\hline & Week $17=11.50 \pm 3.30$ & Data collection week $\times$ sex & 4 & 99.99 & 1.85 & 0.13 \\
\hline & Week $25=18.86 \pm 3.39$ & Data collection week $\times$ cognitive state & 4 & 99.99 & 1.00 & 0.41 \\
\hline & & Sex $\times$ cognitive state & 1 & 39.30 & 4.06 & 0.05 \\
\hline & & Data collection week $\times \operatorname{sex} \times$ cognitive state & 4 & 99.99 & 1.24 & 0.30 \\
\hline \multirow[t]{7}{*}{ Daytime naps } & Baseline $=69.58 \pm 6.84$ & Data collection week & 4 & 105.53 & 0.58 & 0.68 \\
\hline & Week $3=65.09 \pm 7.00$ & Sex & 1 & 40.54 & 1.03 & 0.32 \\
\hline & Week $9=68.16 \pm 7.12$ & Cognitive state & 1 & 40.54 & 0.77 & 0.39 \\
\hline & Week $17=72.80 \pm 7.86$ & Data collection week $\times$ sex & 4 & 105.53 & 0.31 & 0.87 \\
\hline & Week $25=61.14 \pm 7.89$ & Data collection week $\times$ cognitive state & 4 & 105.53 & 0.07 & 0.99 \\
\hline & & Sex $\times$ cognitive state & 1 & 40.54 & 0.12 & 0.73 \\
\hline & & Data collection week $\times$ sex $\times$ cognitive state & 4 & 105.53 & 0.59 & 0.67 \\
\hline
\end{tabular}

Statistically significant $(p<0.05)$ results are shown in bold. 
Table 3

LMEM results for the study's secondary aim, by outcome measure

\begin{tabular}{|c|c|c|c|c|c|c|}
\hline Outcome & $\begin{array}{l}\text { Estimated Mean } \pm \text { SEM, } \\
\text { by week }\end{array}$ & Main effect/interaction & df & Error & $F$ & $p$ \\
\hline \multirow[t]{7}{*}{ Depression (CSDD) } & Baseline $=11.36 \pm 0.74$ & Data collection week & 4 & 97.05 & 15.26 & $<0.001$ \\
\hline & Week $3=7.47 \pm 0.74$ & Sex & 1 & 43.85 & 0.05 & 0.82 \\
\hline & Week $9=6.02 \pm 0.76$ & Cognitive state & 1 & 43.85 & 3.23 & 0.08 \\
\hline & Week $17=5.95 \pm 0.84$ & Data collection week $\times$ sex & 4 & 97.05 & 0.74 & 0.56 \\
\hline & Week $25=4.18 \pm 0.88$ & Data collection week $\times$ cognitive state & 4 & 97.05 & 0.79 & 0.53 \\
\hline & & Sex $\times$ cognitive state & 1 & 43.85 & 0.58 & 0.45 \\
\hline & & Data collection week $\times \operatorname{sex} \times$ cognitive state & 4 & 97.05 & 0.36 & 0.84 \\
\hline \multirow{7}{*}{ Agitation (CMAI) } & Baseline $=47.10 \pm 1.98$ & Data collection week & 4 & 94.91 & 10.41 & $<0.001$ \\
\hline & Week $3=40.89 \pm 1.98$ & Sex & 1 & 42.15 & 0.84 & 0.36 \\
\hline & Week $9=37.54 \pm 2.01$ & Cognitive state & 1 & 42.15 & 1.13 & 0.30 \\
\hline & Week $17=36.80 \pm 2.13$ & Data collection week $\times$ sex & 4 & 94.91 & 1.90 & 0.12 \\
\hline & Week $25=35.33 \pm 2.23$ & Data collection week $\times$ cognitive state & 4 & 94.91 & 2.70 & 0.04 \\
\hline & & Sex $\times$ cognitive state & 1 & 42.15 & 0.003 & 0.96 \\
\hline & & Data collection week $\times$ sex $\times$ cognitive state & 4 & 94.91 & 0.82 & 0.52 \\
\hline \multirow[t]{7}{*}{ Quality of life (MDS-ADL) } & Baseline $=12.38 \pm 1.25$ & Data collection week & 4 & 89.89 & 0.96 & 0.43 \\
\hline & Week $3=12.78 \pm 1.25$ & Sex & 1 & 42.71 & 0.04 & 0.85 \\
\hline & Week $9=11.98 \pm 1.26$ & Cognitive state & 1 & 42.71 & 0.81 & 0.37 \\
\hline & Week $17=12.89 \pm 1.30$ & Data collection week $\times$ sex & 4 & 89.89 & 3.23 & 0.02 \\
\hline & Week $25=13.41 \pm 1.32$ & Data collection week $\times$ cognitive state & 4 & 89.89 & 1.20 & 0.32 \\
\hline & & Sex $\times$ cognitive state & 1 & 42.71 & 0.12 & 0.73 \\
\hline & & Data collection week $\times$ sex $\times$ cognitive state & 4 & 89.89 & 1.27 & 0.29 \\
\hline
\end{tabular}

CSDD, Cornell Scale for Depression in Dementia; CMAI, Cohen-Mansfield Agitation Inventory; LMEM, Linear mixed-effects model; MDS-ADL, Minimum Data Set Activities of Daily Living Scale. Statistically significant $(p<0.05)$ results are shown in bold.

\section{Subjective assessment of agitation (CMAI)}

The LMEM revealed a significant main effect of data collection week and a significant interaction between data collection week and cognitive state (see Table 3). The lighting intervention was more effective for the group diagnosed with severe dementia compared to those in the mild-moderate group. For the mild-moderate group, CMAI scores were significantly lower at week 9 compared to baseline; for the severe group, CMAI scores were significantly lower at weeks 3, 9, 17, and 25 compared to the baseline week scores (Fig. 3B, Supplementary Table 5). Lower CMAI scores indicate reduced frequency of agitation.

\section{Subjective assessment of quality of life (MDS-ADL)}

The LMEM revealed a significant interaction between data collection week and sex for MDS-ADL (see Table 3). Post hoc comparisons did not, however, revealed any significant effects. In general, males had higher scores and their scores increased over the course of the study, while females scores remained lower and more steady. A higher MDS-ADL score is associated with greater dependence in the performance of personal care.

\section{DISCUSSION}

In the previously published, short-term phase of this study [31], we demonstrated that an all-day TLI designed to maximally affect the circadian system reduced sleep disturbances and symptoms of depression and agitation in patients with ADRD, which is consistent with other studies [28, 49-51]. The results of the present within-subjects field study demonstrate that a long-term exposure to the TLI improved subjective and objective measures of sleep, as well as subjective measures of depression and agitation, in participants with moderate to late-stage ADRD.

After the 6-month TLI, participants' mean PSQI scores were significantly reduced from greater than 11 at baseline to close to 5 (see Table 2A), the latter value being virtually at the threshold for the absence of sleep disturbances (scores $<5$ indicate no sleep disturbances), with a very large effect size (see Supplementary Table 3). It is noteworthy that PSQI scores continuously declined over the course of the study, consistent with our previous studies [26, 32]. Thus, long-term daytime light exposures appear to be required to observe positive outcomes for persons with dementia, perhaps explaining why so many past studies have not found an improvement in sleep disturbances with a lighting intervention [52]. 
A

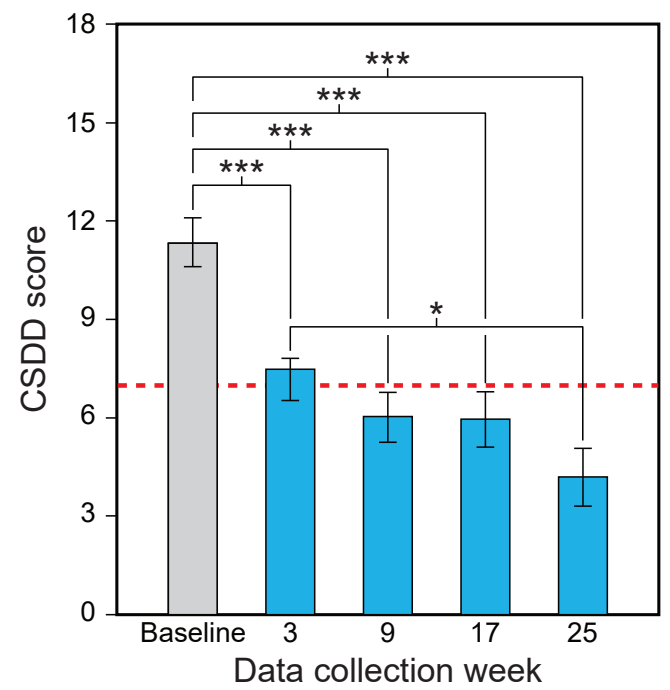

B

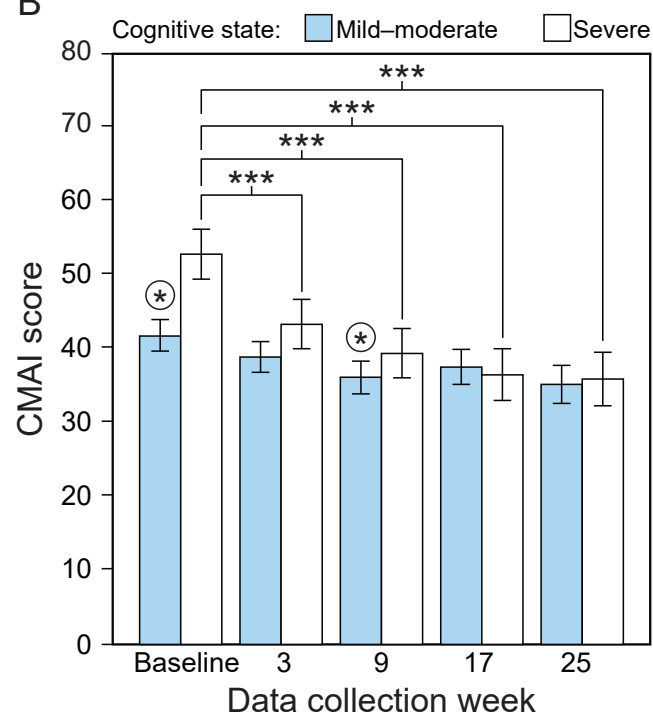

Fig. 3. Results for the study's secondary aim, showing the statistically significant effect of data collection week for CSDD (A) and the significant interaction between data collection and cognitive state for CMAI (B). Mean CSDD scores were significantly lower at weeks 3, 9,17 , and 25 compared to baseline, and significantly lower at week 25 compared to week 3 . Lower CSDD scores indicate less depression and scores $>7$ (dashed line) indicate the presence of depression. CMAI scores for the mild-moderate group were significantly lower only at week 9 compared to baseline (circled asterisks). CMAI scores for the severe group, on the other hand, were significantly lower at weeks $3,9,17$, and 25 compared to baseline. Lower CMAI scores indicate reduced frequency of agitation. The values represent estimated means and the error bars indicate SEM. CSDD, Cornell Scale for Depression in Dementia; CMAI Cohen-Mansfield Agitation Inventory; ${ }^{*} p<0.05$; $* * * p<0.001$.

With respect to objective measures of rest-activity rhythms and sleep, it was interesting to observe that, contrary to expectations, participants with mild-moderate dementia were more disrupted (i.e., greater fragmentation of the rest-activity rhythm, as exhibited by higher IV scores) than those with severe dementia, perhaps because dementia patients become more sedentary as the disease progresses. The IV ratio quantifies the frequency and the extent of transitions between periods of rest and activity, so a person who is more sedentary will exhibit fewer transitions between rest and activity. Contrary to the results of a study by Van Someren et al. [49], who exposed ADRD patients to light for 4 consecutive weeks, our results did not show any effect of the intervention on measures of rest-activity patterns. These differences may be attributable to the length of our studies or the differences in the severity of dementia exhibited by participants in each of the studies.

Sleep efficiency improved compared to baseline, but this effect was gradual, with the greatest improvements being observed at data collection weeks 17 and 25. Sleep onset latency also showed a gradual pattern with a significant, sharp decrease in sleep onset latency at week 9, but it slowly increased and was marginally greater than baseline levels in week 25. Sleep onset latency was also greater in those with severe dementia (see Supplementary Table 3). Together, these results suggest that changes in actigraphic measures of sleep take a longer time to register than changes observed in subjective measures and, in some cases (e.g., sleep onset latency), may not persist for a long period of time.

Despite studies showing that subjective scales are not as reliable as actigraphy data [53-55], the present results suggest that proxy data from caregivers may detect early signs of behavioral or social improvements that are not necessarily readily registered by actigraphy. In this regard, a 2014 Cochrane review [52] of the effectiveness of light therapy for treating dementia symptoms reported results from three studies [56-59] showing that 2-10 weeks of treatment did not significantly increase sleep efficiency values (measured via wrist actigraphy), which led the review's authors to conclude that light therapy is ineffective for improving that outcome in persons with ADRD. Our results suggest that a longer exposure duration can be effective for improving rest-activity consolidation and sleep in this population; indeed, the longer the duration of the 
TLI, the greater the improvement in some measures of sleep.

The present results did not reveal any other significant effects of the TLI on objective measures of sleep. Actual sleep time increased by about 16 minutes between baseline and week 25 , but the increase was not statistically significant. These improvements are similar to those found by McCurry et al. [58] and Riemersma van de Lek et al. [28], who showed statistically nonsignificant increases in sleep duration of 27 and 36 minutes, respectively, after a 6-month lighting intervention.

With respect to the study's secondary aim, the present results showed a significant improvement in depression scores starting at week 3 and continuing over the course of the protocol. At baseline, the participants' mean CSDD score of 11 was within the range for mild depression (8-12 points), but after 3 weeks of exposure to the TLI the mean score was reduced to $<7$ (no depression) and at the end of week 25 was further reduced to 4 (see Fig. 4A). These results are not consistent with those of Hickman et al., who did not see significant effects from 3 -week morning, evening, or all-day light treatments on depressive symptoms [60]. Those investigators reported a strongest effect of morning light that was more favorable in women than in men. In the present study, the females outnumbered the males by about $30 \%$, which may explain why we observed positive results in the CSDD outcome for the TLI. These results are, however, consistent with those of Riemersma van de Lek et al. [28], who showed an significant improvement of 1.5 points in CSDD scores at 3.5-year follow up (or a relative 19\% improvement) and an improvement of 1.8 points at 1.5-year follow up. In the present study, there was an improvement of 7 points in CSDD scores, which is remarkably higher.

Finally, with respect to agitation scores, a CMAI score $>45$ is considered to indicate clinically significant agitation. The participants' mean baseline score of 47 was significantly reduced to 35 after exposure to the 6-month intervention. Consistent with our previous study [31], a reduction in agitation with the TLI was observed early in the treatment (i.e., at week 9). The interaction between lighting intervention and cognitive state showed that the intervention was more effective among those with severe dementia, who also had a higher mean CMAI score at the beginning of the study. Riemersma van de Lek et al. [28] did not find a significant effect of light on CMAI scores, but they did observe a 3.9-point reduction in CMAI scores when combining light and oral melatonin. Of course, their results may be explained by the longer length of their study. It should be noted that a systematic review of the clinical effectiveness of light therapy for managing agitation in persons with ADRD concluded that none of the examined studies, which delivered light therapy for periods of 30 minutes to 2 hours per day over the course of 7 days to 10 weeks, revealed a positive effect [61]. This lack of positive effect, again, underscores the importance of controlling the stimulus and lengthening the treatment duration to obtain positive results.

Although the present study was not designed to compare differences in sex and cognitive state with respect to sleep and rest-activity parameters, our data showed some other interesting interactions. In general, males exhibited greater PSQI scores, lower actual sleep time percentages, lower actual sleep times, and greater actual wake time percentages. Future studies should further investigate sex differences in sleep measures. Participants with severe dementia also exhibited less-disrupted rest-activity rhythms (demonstrated by their significantly lower IV scores), reduced actual sleep times, and greater impact from the TLI on agitation (CMAI) scores. It is reasonable to assume that persons with a higher level of dementia may have greater difficulty with mobility and tend to maintain a more regular, lessactive pattern over the course of the day. This does not mean, however, that they experience better sleep than those with mild-moderate dementia.

Several possible limitations should be addressed, the most notable perhaps being that the present study was performed in a somewhat heterogeneous group of ADRD patients who are representative of those living in more-controlled environments. But we see this as a strength because the study should be considered a practical clinical trial that, like the one performed by Riemersma-van der Lek and colleagues [28], provides healthcare decision makers with a more realistic set of results. One could argue that the present study fits into the category of pragmatic clinical trial, wherein the efficacy of interventions such as the ones used in the present study are tested in real-life settings.

One might also see a significant limitation in the study's use of professional caregivers to answer the questionnaires, thereby potentially introducing an element of bias. We chose to collect proxy data because all of the participants were moderately to severely demented and thus limited in their ability to provide self reports. Caregivers' responses, however, did not always favor the outcomes associated with the 

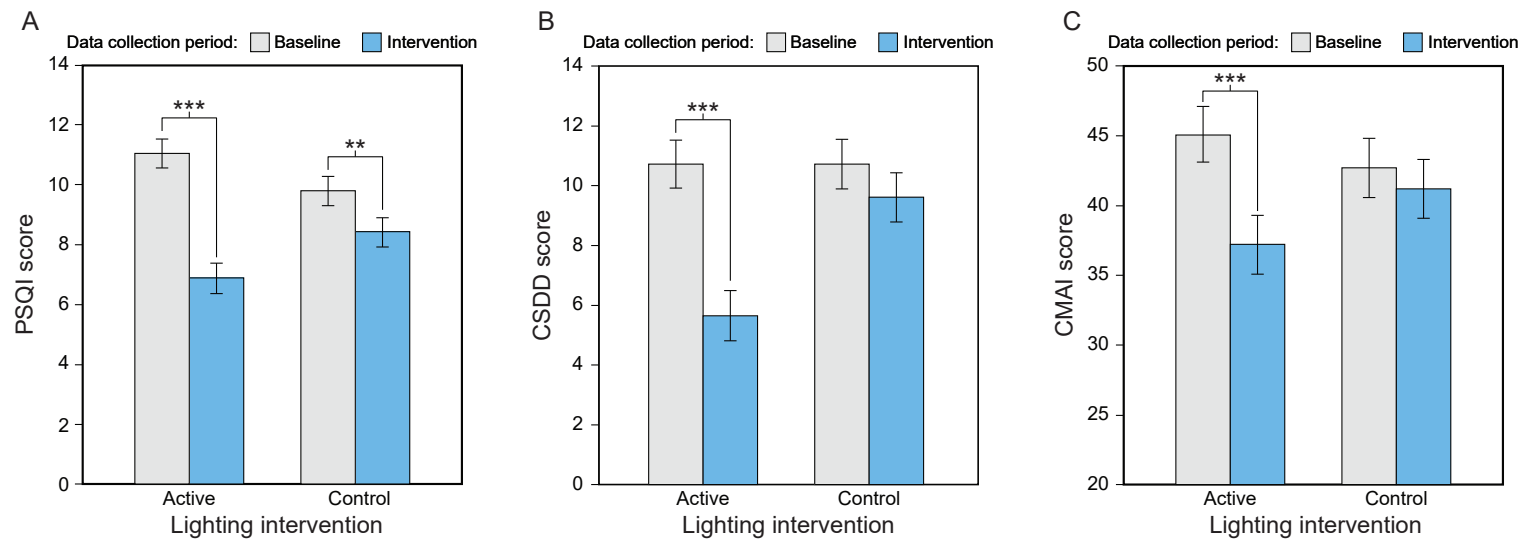

Fig. 4. Results from post hoc analyses comparing the present results with a control group used data from our previous short-term study (see Supplementary Table 6) [31]. (A) PSQI scores at baseline were not significantly different, but they were significantly different after both the active and the control intervention. The effect size for the difference in the active intervention was much larger compared to the control. (B) Although CSDD scores were not significantly different between the active and control intervention at baseline, only the active intervention was significantly different from baseline. (C) Although CMAI scores were not significantly different between the active and control intervention at baseline, only the active intervention was significantly different from baseline. The error bars represent SEM. PSQI, Pittsburgh Sleep Quality Index; CMAI, Cohen-Mansfield Agitation Inventory; CSDD, Cornell Scale for Depression in Dementia; $* * p<0.01$; $* * * p<0.001$.

Table 4

LMEM results for comparison between the present study and our previously published study [31], by outcome measure

\begin{tabular}{|c|c|c|c|c|c|c|c|c|}
\hline Outcome & & $\begin{array}{l}\text { timated Mean } \\
\text { lighting inter } \\
\text { data collecti }\end{array}$ & $\begin{array}{l}\text { EM, } \\
\text { tion } \\
\text { eriod }\end{array}$ & Main effect/interaction & $\mathrm{df}$ & Error & $F$ & $p$ \\
\hline \multirow[t]{3}{*}{$\overline{\text { Sleep quality (PSQI) }}$} & Active & Baseline & $11.04 \pm 0.48$ & Lighting intervention & 1 & 89.22 & 3.78 & 0.06 \\
\hline & & Intervention & $6.88 \pm 0.50$ & Data collection period & 1 & 85.78 & 30.38 & $<0.001$ \\
\hline & Control & Baseline & $9.80 \pm 0.49$ & Lighting intervention $x$ & 1 & 85.78 & 12.44 & 0.001 \\
\hline \multirow[t]{3}{*}{ Depression (CSDD) } & Active & Baseline & $10.72 \pm 0.80$ & Lighting intervention & 1 & 87.65 & 0.06 & 0.811 \\
\hline & & Intervention & $5.65 \pm 0.84$ & Data collection period & 1 & 84.80 & 56.69 & $<0.001$ \\
\hline & Control & $\begin{array}{l}\text { Baseline } \\
\text { Intervention }\end{array}$ & $\begin{array}{l}10.73 \pm 0.83 \\
9.61 \pm 0.83\end{array}$ & $\begin{array}{l}\text { Lighting intervention } \times \\
\text { data collection period }\end{array}$ & 1 & 84.80 & 14.20 & $<0.001$ \\
\hline \multirow[t]{4}{*}{ Agitation (CMAI) } & Active & Baseline & $45.06 \pm 2.05$ & Lighting intervention & 1 & 87.44 & 0.09 & 0.77 \\
\hline & & Intervention & $37.21 \pm 2.11$ & Data collection period & 1 & 82.79 & 19.53 & $<0.001$ \\
\hline & Control & Baseline & $42.70 \pm 2.11$ & Lighting intervention $\times$ & 1 & 82.79 & 9.01 & 0.004 \\
\hline & & Intervention & $41.20 \pm 2.11$ & data collection period & & & & \\
\hline
\end{tabular}

PSQI, Pittsburgh Sleep Quality Index; CSDD, Cornell Scale for Depression in Dementia; CMAI, Cohen-Mansfield Agitation Inventory; LMEM, Linear mixed-effects model. Statistically significant $(p<0.05)$ results are shown in bold.

TLI. For example, the MDS-ADL scores remained stable (see Table 3), which would be expected given that physical functioning of older adults is influenced by multiple comorbid factors other than just sleep quality. Another possible limitation was the use of the PSQI as a subjective scale to assess sleep disturbances because one of the components in the PSQI relates to the partner or roommate. It should be emphasized, however, that the PSQI instrument provides an opportunity for raters to respond that the participant does not have a partner or roommate, which was always the case among the single-room participants in our study. Moreover, we also evaluated the scores for PSQI components 1-4 only (sleep-related components) and observed the same results; therefore, we do not see a reason why the raters' responses would have skewed their evaluations of the participants' sleep disturbances.

More importantly, the present results demonstrate that any element of bias that might have been introduced by the caregivers' questionnaire responses could not possibly account for the strong effect size 
observed in the post hoc analyses (see Supplementary Tables 3-5). Moreover, among the few studies that have collected longitudinal data on the effects of light on sleep and behavior in ADRD patients for as long as 6 months, one study showed participants' depression scores (CSDD) increasing from 7.6 to 9.3 and their agitation scores (CMAI) increasing from 45 to 47 during exposure to that study's control condition (no additional lighting) [28]. The trend for these measures, in other words, is typically toward decrements rather than improvements in the absence of an effective lighting intervention.

Another limitation of the study lies in our employing a within-subjects experimental design that compared the effects of the TLI with baseline measures. Given the protocol's length, however, employing a crossover design would have been too burdensome on the participants, facility staff, and researchers. It should be stressed that these results are consistent with our previously published, recent randomized, crossover, placebo-controlled study [31]. In this related short-term study [31], the same outcome measures and procedures were employed, with the addition of a placebo lighting intervention (the control) that was administered in a randomized, crossover, within-subjects protocol. (The control delivered a $\mathrm{CS}<0.1$, which is below the threshold for activation of the circadian system.) While that study's results showed a small, but statistically nonsignificant effect of the placebo condition, the results from the active lighting intervention $(\mathrm{CS}=0.3)$ were significantly different from the baseline values with large effect sizes.

Nonetheless, to confirm that our present results were not an artifact of the study's experimental design, we performed secondary analyses using data collected for the same outcome measures in our previous study [31]. Briefly, that 14-week randomized, placebo-controlled, crossover design clinical trial administered an all-day active or control TLI to 46 patients with ADRD in eight long-term care facilities for two 4-week periods (separated by a 4week washout). Baseline data (weeks 1 and 10) were collected prior to each 4-week lighting intervention period. We used these two sets of baseline data as a control group (no lighting intervention was employed during these 2 weeks) and compared them to data from week 1 (baseline, no lighting intervention) and week 9 (active lighting intervention) of the present study (Supplementary Fig. 1). The hypothesis was that there would be no difference between week 1 of both studies, but that there would a significant difference between weeks 9 (present study where TLI was administered from weeks 2 to 25 ) and 10 (the previous study's second baseline data collection week). Figure 4, Table 4, and Supplementary Table 6 show the comparative results of the PSQI, CSDD, and CMAI outcome measures for both studies. As expected, statistically significant interactions were found for these outcomes. While sleep disturbance, depression, and agitation scores were not significantly different between the two groups at baseline, these scores were significantly lower in the active lighting intervention group but not in the control at weeks 9 (present study) and 10 (previous study). With respect to actigraphic measures, IS, sleep efficiency, and actual sleep times were significantly higher and sleep onset latency was significantly shorter during the intervention compared to baseline. IV was significantly lower in the intervention compared to the control condition. These results suggest that, in a between-subjects analyses and compared to a control group, the intervention was similarly effective for improving study outcomes.

Lastly, the present study was limited by not collecting markers of circadian entrainment nor distinguishing between the participants diagnosed with Alzheimer's disease from those experiencing related dementias to test the effectiveness of the TLI between groups with overlapping symptoms. The infeasibility of obtaining core body temperature minimum [62] or dim light melatonin onset [63] data (both well-stablished markers of circadian phase) in this population precluded assessments of circadian entrainment. Future studies could be designed to collect, perhaps, urinary melatonin as a surrogate measure of circadian entrainment. A vision test was not performed, but we excluded those known to have blindness, macular degeneration, or cataracts, which would have most affected the participants' response to the TLI.

The present results extend from our short-term field studies [26, 31, 32] to demonstrate, in a long-term study, that light tailored to maximally affect the circadian system, especially when carefully delivered and measured in the field, can continuously improve sleep quality, depressive symptoms, and agitation in patients with ADRD. The TLI was also well tolerated by the participants, which is crucial for the effective delivery of a lighting intervention in real-world applications. Together, these data converge to support the inference that correctly specified, daytime TLIs can lead to positive, clinically important outcomes for persons with ADRD. 


\section{ACKNOWLEDGMENTS}

This research was funded by the National Institute on Aging (grant \# R01AG034157). The clinical trial for the study was registered as "Methodology Issues in a Tailored Light Treatment for Persons with Dementia," https://clinical trials.gov/ct2/show/NCT01816152, Trial No. NCT01816152. The following manufacturers are acknowledged for their provision of in-kind lighting products: GE Current, a Daintree company; GE Lighting; Ketra; and OSRAM Sylvania; Sharp Corporation. Neither the funding agency nor the in-kind contributors had any role in the design, methods, data analysis, or preparation of the manuscript. The authors would like to acknowledge Bridget Bradley, Sharon Lesage, Savana Wemette, Charles Roohan, Martin Overington, Howard Ohlhous, Madison Laks, and Gregory Ward for their technical support in data collection and analyses. David Pedler (Lighting Research Center, Rensselaer Polytechnic Institute) served as the technical editor.

\section{CONFLICT OF INTEREST}

The authors have no conflict of interest to report.

\section{SUPPLEMENTARY MATERIAL}

The supplementary material is available in the electronic version of this article: https://dx.doi.org/ 10.3233/ADR-200212.

\section{REFERENCES}

[1] Alzheimer's Association (2019) 2019 Alzheimer's disease facts and figures. Alzheimers Dement 15, 321-387.

[2] Vitiello MV, Bliwise DL, Prinz PN (1992) Sleep in Alzheimer's disease and the sundown syndrome. Neurology 42, 89-93.

[3] Vitiello MV, Borson S (2001) Sleep disturbances in patients with Alzheimer's disease: Epidemiology, pathophysiology and treatment. CNS Drugs 15, 777-796.

[4] McCurry SM, Teri L (1996) Sleep disturbance in elderly caregivers of dementia patients. Clin Gerontol 16, 51-66.

[5] McCurry SM, Gibbons LE, Logsdon RG, Vitiello MV, Teri L (2009) Insomnia in caregivers of persons with dementia: Who is at risk and what can be done about it? Sleep Med Clin 4, 519-526.

[6] Cohen-Mansfield J, Billig N, Lipson S, Rosenthal AS, Pawlson LG (1990) Medical correlates of agitation in nursing home residents. Gerontology 36, 150-158.

[7] Bliwise DL (1993) Sleep in normal aging and dementia. Sleep 16, 40-81.
[8] Bliwise DL, Hughes M, McMahon PM, Kutner N (1995) Observed sleep/wakefulness and severity of dementia in an Alzheimer's disease special care unit. J Gerontol A Biol Sci Med Sci 50, M303-M306.

[9] Hinton DR, Sadun AA, Blanks JC, Miller CA (1986) Opticnerve degeneration in Alzheimer's disease. $N$ Engl J Med 315, 485-487.

[10] Katz B, Rimmer S, Iragui V, Katzman R (1989) Abnormal pattern electroretinogram in Alzheimer's disease: Evidence for retinal ganglion cell degeneration? Ann Neurol 26, 221225.

[11] Moore RY (1992) The Fourth C.U. Ariëns Kappers Lecture. The organization of the human circadian timing system Prog Brain Res 9, 99-115; discussion 115-117.

[12] Swaab DF, Fliers E, Partiman TS (1985) The suprachiasmatic nucleus of the human brain in relation to sex, age and senile dementia. Brain Res 342, 37-44.

[13] Campbell SS, Kripke DF, Gillin JC, Hrubovcak JC (1988) Exposure to light in healthy elderly subjects and Alzheimer's patients. Physiol Behav 42, 141-144.

[14] Ancoli-Israel S, Kripke DF (1989) Now I lay me down to sleep: The problem of sleep fragmentation in elderly and demented residents of nursing homes. Bull Clin Neurosci 54, 127-132.

[15] Weale RA (1961) Retinal illumination and age. Trans Illum Eng Soc (NY) 26, 95-100.

[16] Turner PL, Mainster MA (2008) Circadian photoreception: Ageing and the eye's important role in systemic health. $\mathrm{Br}$ J Ophthalmol 92, 1439-1444.

[17] Prinz PN, Peskind ER, Vitaliano PP, Raskind MA, Eisdorfer C, Zemcuznikov N, Gerber CJ (1982) Changes in the sleep and waking EEGs of nondemented and demented elderly subjects. J Am Geriatr Soc 30, 86-93.

[18] Satlin A, Teicher MH, Lieberman HR, Baldessarini RJ, Volicer L, Rheaume Y (1991) Circadian locomotor activity rhythms in Alzheimer's disease. Neuropsychopharmacology 5, 115-126.

[19] Shochat T, Martin J, Marler M, Ancoli-Israel S (2000) Illumination levels in nursing home patients: Effects on sleep and activity rhythms. J Sleep Res $\mathbf{9}, 373-379$.

[20] McCleery J, Cohen DA, Sharpley AL (2016) Pharmacotherapies for sleep disturbances in dementia. Cochrane Database Syst Rev, CD009178.

[21] Schroeck JL, Ford J, Conway EL, Kurtzhalts KE, Gee ME, Vollmer KA, Mergenhagen KA (2016) Review of safety and efficacy of sleep medicines in older adults. Clin Ther 38, 2340-2372.

[22] Deschenes CL, McCurry SM (2009) Current treatments for sleep disturbances in individuals with dementia. Curr Psychiatry Rep 11, 20-26.

[23] Ooms S, Ju Y-E (2016) Treatment of sleep disorders in dementia. Curr Treat Options Neurol 18, 40-40.

[24] Picton JD, Marino AB, Nealy KL (2018) Benzodiazepine use and cognitive decline in the elderly. Am J Health Syst Pharm 75, e6-e12.

[25] Pasqualetti G, Tognini S, Calsolaro V, Polini A, Monzani F (2015) Potential drug-drug interactions in Alzheimer patients with behavioral symptoms. Clin Interv Aging 10, 1457-1466.

[26] Figueiro MG, Plitnick BA, Lok A, Jones GE, Higgins P, Hornick TR, Rea MS (2014) Tailored lighting intervention improves measures of sleep, depression, and agitation in persons with Alzheimer's disease and related dementia living in long-term care facilities. Clin Interv Aging $\mathbf{9}$, 1527-1537. 
[27] Figueiro MG (2017) Light, sleep and circadian rhythms in older adults with Alzheimer's disease and related dementias. Neurodegener Dis Manag 7, 119-145.

[28] Riemersma-van der Lek RF, Swaab DF, Twisk J, Hol EM, Hoogendijk WJ, Van Someren EJW (2008) Effect of bright light and melatonin on cognitive and noncognitive function in elderly residents of group care facilities: A randomized controlled trial. J Am Med Assoc 299, 2642-2655.

[29] Thapan K, Arendt J, Skene DJ (2001) An action spectrum for melatonin suppression: Evidence for a novel non-rod, non-cone photoreceptor system in humans. J Physiol 535, 261-267.

[30] Brainard GC, Hanifin JP, Greeson JM, Byrne B, Glickman G, Gerner E, Rollag MD (2001) Action spectrum for melatonin regulation in humans: Evidence for a novel circadian photoreceptor. J Neurosci 21, 6405-6412.

[31] Figueiro MG, Plitnick B, Roohan C, Sahin L, Kalsher M, Rea MS (2019) Effects of a tailored lighting intervention on sleep quality, rest-activity, mood, and behavior in older adults with Alzheimer's disease and related dementias: A randomized clinical trial. J Clin Sleep Med 15, 1757-1767.

[32] Figueiro MG, Hunter CM, Higgins PA, Hornick TR, Jones GE, Plitnick B, Brons JA, Rea MS (2015) Tailored lighting intervention for persons with dementia and caregivers living at home. Sleep Health 1, 322-330.

[33] World Medical Association (2000) World Medical Association Declaration of Helsinki: Ethical principles for medical research involving human subjects. JAMA 284, 3043-3045.

[34] American Psychiatric Association (2000) Diagnostic and Statistical Manual of Mental Disorders, American Psychiatric Association, Arlington, VA.

[35] Folstein MF, Folstein SE, McHugh PR (1975) Mini-mental state: A practical method for grading the cognitive state of patients for the clinician. J Psychiatr Res 12, 189-198.

[36] Saliba D, Buchanan J, Edelen MO, Streim J, Ouslander J, Berlowitz D, Chodosh J (2012) MDS 3.0: Brief interview for mental status. J Am Med Dir Assoc 13, 611-617.

[37] Buysse DJ, Reynolds CF, Monk TH, Berman SR, Kupfer DJ (1989) The Pittsburgh Sleep Quality Index: A new instrument for psychiatric practice and research. Psychiatry Res 28, 193-213.

[38] Dixon JS, Saddington DG, Shiles CJ, Sreevalsan KP, Munro CA, Rosenberg PB (2017) Clinical evaluation of brief cognitive assessment measures for patients with severe dementia. Int Psychogeriatr 29, 1169-1174.

[39] Douglas AB, Bornstein R, Nino-Murcia G, Keenan S, Miles L, Zarcone VPJ, Guilleminault C, Dement WC (1994) The Sleep Disorders Questionnaire 1: Creation and multivariate structure of SDQ. Sleep 17, 160-167.

[40] Walters AS, LeBrocq C, Dhar A, Hening W, Rosen R, Allen RP, Trenkwalder C (2003) Validation of the International Restless Legs Syndrome Study Group rating scale for restless legs syndrome. Sleep Med 4, 121-132.

[41] Rea MS, Figueiro MG, Bullough JD, Bierman A (2005) A model of phototransduction by the human circadian system. Brain Res Rev 50, 213-228.

[42] Rea MS, Figueiro MG, Bierman A, Hamner R (2012) Modelling the spectral sensitivity of the human circadian system. Light Res Technol 44, 386-396.

[43] Rea MS, Figueiro MG (2018) Light as a circadian stimulus for architectural lighting. Light Res Technol 50, 497-510.

[44] Figueiro MG, Hamner R, Bierman A, Rea MS (2013) Comparisons of three practical field devices used to measure personal light exposures and activity levels. Light Res Technol 45, 421-434.
[45] DiLaura D, Houser K, Mistrick R, Steffy G eds. (2011) IES Lighting Handbook: Reference and Application, Illuminating Engineering Society of North America, New York, NY.

[46] Alexopolous GS, Abrams RC, Young RC, Shamoian CA (1988) Cornell Scale for Depression in Dementia. Biol Psychiatry 23, 271-284.

[47] Cohen-Mansfield J, Marx MS, Rosenthal AS (1989) A description of agitation in a nursing home. J Gerontol 44, M77-M84.

[48] Galasko D, Bennett D, Sano M, Ernesto C, Thomas R, Grundman M, Ferris S (1997) An inventory to assess activities of daily living for clinical trials in Alzheimer's disease. The Alzheimer's Disease Cooperative Study. Alzheimer Dis Assoc Disord 11 Suppl 2, S33-S39.

[49] Van Someren EJW, Kessler A, Mirmiran M, Swaab DF (1997) Indirect bright light improves circadian rest-activity rhythm disturbances in demented patients. Biol Psychiatry 41, 955-963.

[50] Mishima K, Hishikawa Y, Okawa M (1998) Randomized, dim light controlled, crossover test of morning bright light therapy for rest-activity rhythm disorders in patients with vascular dementia and dementia of Alzheimer's type. Chronobiol Int 15, 647-654.

[51] Yamadera H, Ito T, Suzuki H, Asayama K, Ito R, Endo S (2000) Effects of bright light on cognitive and sleep-wake (circadian) rhythm disturbances in Alzheimer-type dementia. Psychiatry Clin Neurosci 54, 352-353.

[52] Forbes D, Blake CM, Thiessen EJ, Peacock S, Hawranik P (2014) Light therapy for improving cognition, activities of daily living, sleep, challenging behaviour, and psychiatric disturbances in dementia. Cochrane Database Syst Rev 2, CD003946.

[53] Grandner MA, Kripke DF, Yoon I-Y, Youngstedt SD (2006) Criterion validity of the Pittsburgh Sleep Quality Index: Investigation in a non-clinical sample. Sleep Biol Rhythms 4, 129-139.

[54] Buysse DJ, Hall ML, Strollo PJ, Kamarck TW, Owens J, Lee L, Reis SE, Matthews KA (2008) Relationships between the Pittsburgh Sleep Quality Index (PSQI), Epworth Sleepiness Scale (ESS), and clinical/polysomnographic measures in a community sample. J Clin Sleep Med 4, 563-571.

[55] Landry GJ, Best JR, Liu-Ambrose T (2015) Measuring sleep quality in older adults: A comparison using subjective and objective methods. Front Aging Neurosci 7, 166-166.

[56] Dowling GA, Graf CL, Hubbard EM, Luxenberg JS (2007) Light treatment for neuropsychiatric behaviors in Alzheimer's disease. West J Nurs Res 29, 961-975.

[57] Dowling GA, Mastick J, Hubbard EM, Luxenberg JS, Burr RL (2005) Effect of timed bright light treatment for rest-activity disruption in institutionalized patients with Alzheimer's disease. Int J Geriatr Psychiatry 20, 738-743.

[58] McCurry SM, Pike KC, Vitiello MV, Logsdon RG, Larson EB, Teri L (2011) Increasing walking and bright light exposure to improve sleep in community-dwelling persons with Alzheimer's disease: Results of a randomized, controlled trial. J Am Geriatr Soc 59, 1393-1402.

[59] Nowak L (2008) The effect of timed blue-green light on sleep-wake patterns in women with Alzheimer's disease (thesis). Advisor: Jean E. Davis. College of Nursing, Wayne State University, Ann Arbor.

[60] Hickman SE, Barrick AL, Williams CS, Zimmerman S, Connell BR, Preisser JS, Mitchell CM, Sloane PD (2007) The effect of ambient bright light therapy on depressive 
symptoms in persons with dementia. J Am Geriatr Soc 55, 1817-1824.

[61] Livingston G, Kelly L, Lewis-Holmes E, Baio G, Morris S, Patel N, Omar RZ, Katona C, Cooper C (2014) A systematic review of the clinical effectiveness and cost-effectiveness of sensory, psychological and behavioural interventions for managing agitation in older adults with dementia. Health Technol Assess 18, 1-226, v-vi.

[62] Khalsa SBS, Jewett ME, Duffy JF, Czeisler CA (2000) The timing of the human circadian clock is accurately represented by the core body temperature rhythm following phase shifts to a three-cycle light stimulus near the critical zone. J Biol Rhythms 15, 524-530.

[63] Pandi-Perumal SR, Smits M, Spence W, Srinivasan V, Cardinali DP, Lowe AD, Kayumov L (2007) Dim light melatonin onset (DLMO): A tool for the analysis of circadian phase in human sleep and chronobiological disorders. Prog Neuropsychopharmacol Biol Psychiatry 31, 1-11. 